\title{
Pandemic-stage propagation dynamics in South Africa suggest pre-existing cross-reactive protection against severe Covid-19
}

Francois Engelbrecht ( $\square$ Francois.Engelbrecht@wits.ac.za )

University of the Witwatersrand https://orcid.org/0000-0002-9189-6556

Shabir Madhi

University of the Witwatersrand

Robert Scholes

University of the Witwatersrand

Article

Keywords: propagation dynamics, COVID-19, pre-existing cross-reactive immunity

Posted Date: April 15th, 2021

DOI: https://doi.org/10.21203/rs.3.rs-394828/v1

License: (c) (1) This work is licensed under a Creative Commons Attribution 4.0 International License.

Read Full License 


\title{
Pandemic-stage propagation dynamics in South Africa suggest pre-existing cross-reactive protection against severe Covid-19
}

\author{
Francois A. Engelbrecht ${ }^{*}, 1,2$, Shabir A. Madhi ${ }^{3,4}$ and Robert J. Scholes ${ }^{1,2}$
}

1. Global Change Institute, University of the Witwatersrand, South Africa

2. Covid-19 Environmental Reference Group, South Africa

3. South African Medical Research Council Vaccines and Infectious Diseases Analytics Research Unit (VIDA), University of the Witwatersrand, South Africa
4. African Leadership in Vaccinology Expertise, University of the Witwatersrand, South Africa

\begin{abstract}
Early in the Covid-19 outbreak, it was speculated that pre-existing cross-reactive immunity from prior BCG vaccination or exposure to common cold human coronaviruses might confer some protection against Covid-19. Following a year of circulation of SARS-CoV-2 through the world, epidemiological dynamics allow a test of this hypothesis. A dynamic epidemiological model was fitted to the Covid-19 attributed 'excess deaths' in South Africa, a country with a long-standing BCG vaccination program and where social-economic circumstances potentially result in frequent exposure to common cold coronaviruses. We show that Covid-19 propagation dynamics in South Africa are consistent with an initially fully susceptible population (no prior cross-immunity protecting against infection), but this requires assuming values of the infection fatality rate (IFR) below or at the lower plausible range $(0.31$ to 0.53$)$ of internationally reported values. This suggests that some form of pre-existing protection against severe Covid-19 may exist in South Africa. The observed disease propagation dynamics can be explained by both long-lasting immunity and various scenarios of reinfection in the presence of the more transmissible B.1.351 variant, which is also relatively resistant to antibodies induced by infections of prototype SARSCoV-2. All scenarios of reinfection also require assuming a low IFR in order to replicate the observed attributes of the outbreak.

\section{Introduction}

In the early stages of the Covid-19 pandemic it was suggested that population-wide vaccination programs such as for Bacille Calmette-Guérin (BCG) vaccines may have conferred trainedimmunity to SARS-CoV-2 (O'Neill and Netea, 2020), but later assessments provide evidence to the contrary (Lindestam Arlehamna et al., 2020; Wassenaar et al., 2020). It has also been suggested that previous exposure to common cold cornaviruses could result in cross-reactive Tlymphocyte immunity to SARS-CoV-2 (Braun et al., 2020; Sette and Crotty, 2020). These studies indicated T-cell reactivity to SARS-CoV-2 in more than $30 \%$ of individuals not previously exposed to SARS-CoV-2 (Braun et al,, 2020; Grifoni et al., 2020), however, the clinical relevance of this reactivity is unknown (Sette and Crotty, 2020). Despite limited testing on the African continent, it has been assessed that the burden of Covid-19 in the early stages of the pandemic in African countries was less severe than expected (Mbow et al., 2020, Nature, 2020; ScienceMag 2020; Uoga et al., 2021). This has fueled the notion that greater exposure to common cold coronaviruses in high population density, low-income settings may provide cross-reactive protection to Covid-19 (Mbow et al., 2020; Uoga et al., 2021).
\end{abstract}


In South Africa, millions of people live in crowded conditions and there is a long-standing BCG vaccination program. Hence, South Africa is well placed to investigate whether widespread crossimmunity against COVID-19 exist in the population. The time-course of Covid-19 reported cases in South Africa is shown in Figure 1. With a testing rate of approximately 85 per 1000 population during the course of the first wave, the SARS-CoV-2 positivity rates in suspected cases exceeded $25 \%$ at the peak of the first wave (Our world in data, 2021), suggesting that the recorded number of Covid-19 cases and deaths was likely underestimated, as in most other countries (Li et al., 2020).

The first cases of Covid-19 were detected in March 2020. Propagation of the disease was initially attenuated by a national lockdown, among the strictest in the world (Supplementary Table 1; Stiegler and Bouchard, 2020). In May and June, some non-pharmacological interventions (NPI), particularly permission for mass gatherings and use of public transport, were progressively relaxed, and by June 2020 cases started to surge (Garba et al., 2020; Figure 1). Covid-19 cases peaked in mid-July (NICD, 2020a; Figure 1), with medical infrastructure and services severely tested but generally not overwhelmed. By mid-August, recorded Covid-19 cases, hospitalizations and deaths had all dropped by $80 \%$ relative to the peak (Bradshaw et al., 2020; NICD 2020a, 2020b; Figure 1), and in September a period of 'slow burn' in infections signified a new phase in the progression of the outbreak (Figure 1). Serological testing in selected communities in the Western Cape Province of South Africa suggested 35-45\% of people had been infected with SARS-Cov2 by August 2020 (Hsiao et al., 2020). This is far lower than the threshold range for classical herd immunity to set in, as determined by the plausible range of the basic reproduction number $\left(\mathrm{R}_{0}\right)$ for Covid-19 (Britton et al., 2020; Fontanet and Cauchemez, 2020), and associated range in final epidemic size (Miller, 2012, Meehan et al., 2020). The simplest hypothesis for the first wave peaking below the classical herd immunity threshold is evolving community resistance with the high force of infection, coupled with partial adherence to NPIs (despite return to lower official levels), transiently reducing the effective reproductive rate to below 1.0.

At the end of October 2020 a resurgence of Covid-19 started in the Eastern Cape province and swiftly spread to the Western Cape and KwaZulu-Natal, reaching Gauteng and other interior provinces by December 2020 (Figures 1 and 2). The resurgence exceeded the number of deaths recorded during the first wave (Figure 2), and in December 2020 and January 2021 threatened to overwhelm hospitals and medical infrastructure (NICD, 2020b). There are two plausible explanations for the resurgence of Covid-19: further relaxations in NPI (particularly unrestricted mass gatherings) (Supplementary Table 1, Figure 1); and/or the emergence of the B.1.351 (or N501Y.V2) variant. Based on a modelling assessment undertaken in the United Kingdom, the B.1.1.7 lineage that shares the common N501Y mutation in the spike protein is $40-80 \%$ more transmissible than earlier variants (Volz et al., 2020). Furthermore, the neutralizing antibody activity induced by infection from ancestry SARS-CoV-2 variants has less than a tenth of the potency against viruses with the key mutations of the immundominant epitopes identified in the B.1.351 variant (Greaney et al., 2020; Wibmer et al., 2021). This variant, by February 2021, was the cause of $90 \%$ of all Covid-19 cases in South Africa (Moyo-Gwete et al., 2021). The second wave of infections was in decline across all South African provinces by mid-January 2021, in the presence of enhanced NPI implemented by the South African government in late December 2020 (Figures 1 and 2; Supplementary Table 1).

We use a compartment model of the Covid-19 pandemic in South Africa to explore the propagation dynamics of the disease in the populous provinces of Gauteng, the Western Cape and Eastern Cape. In particular, we test whether the observed dynamics are consistent with the pre-existence of widespread cross-reactive immunity (here defined broadly, to include both 
immunity from earlier exposure to common cold coronaviruses and/or trained immunity derived from BCG and other vaccines) protecting against infection and/or severe Covid-19. We also explore the extent to which Covid-19 reinfections, either because of the B.1.351variant overcoming disease-induced immunity by earlier variants, or because of immunity being gradually lost over a period of months, are consistent with the observed propagation dynamics of the disease. We proceed to project the evolution of the disease in South Africa for these different scenarios, as a way to test the various hypotheses. The findings are interpreted within the context of COVID-19 vaccination programs being started in low and middle income countries such as South Africa.

\section{Results}

\section{Gauteng Province}

Modelled infection curves inversed from excess deaths (Methods) in the Gauteng Province of South Africa, assuming an IFR of 0.31, are shown in black lines in Figure 3 for the scenarios of disease-induced immunity lasting perpetually (panel a), 12 months (panel b) and 6 months (panel d). A scenario where immunity generally lasts 12 months, but where an abrupt loss of immunity in $50 \%$ of the population occurred in late November 2020 in association with the emergence of the B.1.351 variant (Methods), is portrayed in panel d. The reconstructed infection curves span the period 1 March 2020 to 7 March 2021 (Methods). An IFR of 0.31 is well below the internationally estimated plausible range of the IFR, of 0.53 to 0.82 (Meyerowitz-Katz and Merone, 2020). For all cases, the modelled number of infections display the key features of the reported cases and deaths, and excess deaths in Gauteng: a first wave peaking in early July 2020, followed by about three months of low numbers of infections between September and November, and a second wave of amplitude similar to the first (Figure $2 \mathrm{c}$ and g), reaching its peak in early January 2021.

For all the scenarios considered, the number of infections need to be about $26 \%$ of the population by the end of August (representing the end of the first wave; Supplementary Table 2.1), in order for the simulations to reproduce the corresponding number of accumulated excess deaths. Serological surveys indicate about $25 \%$ of the Gauteng population infected by the end of August 2020 (Portia Mutevedzi - MRC, personal communication). The estimated value of $\mathrm{R}_{0}$ ' (the basic reproduction number as modified by NPI, Methods) for the 6-week period preceding the peak of the first wave is close to 1.1 for all the cases considered (Supplementary Table 3.1) (this is because of almost equal fractions of the population being susceptible across the various scenarios during this time). By 7 March, post the second wave, the simulations suggest that the fraction of the total population that had been infected in the first plus second wave is about $51 \%$ (Suppementary Table 2.1). The findings of post second wave seroprevalance surveys are not available for comparison as yet.

The reconstructed values for $\mathrm{R}_{0}$ ' for the second wave range between 2.6 and 3.3 across the various scenarios of reinfection (Supplementary Table 3.1). This range of numerical estimations is a function of the different sizes of the pools of susceptible individuals available at the onset of and during the second wave for the various scenarios under consideration (Methods). The substantial increase in transmissibility from the first wave to the second, estimated to range between 136 and $175 \%$, is likely due to the combined effect of reduced compliance to NPI recommendations (both in terms of rules, and their observance) during the December 2020 vacation period and the arrival of the B.1.351variant in Gauteng in late November 2020. 
Substantially lower values of the IFR in Gauteng province represent a contradiction that begs an explanation. An assumed IFR of 0.2 yields a total of $41 \%$ of the population infected by the end of August (that is, during the first wave; Supplementary Table 2.1), but serological testing suggests a lower percentages (Portia Mutevedzi - MRC, personal communication). The accumulated infections are modelled to be about $80 \%$ of the population by 7 March 2021 (Supplementary Table 2.1).

For an IFR of 0.53 , which is the lower limit of the internationally estimated range (MeyerowitzKatz and Merone, 2020), accumulated infections are simulated to be $15 \%$ by the end of the first wave and $30 \%$ by the end of the second wave (Supplementary Table Table 2.1). The post first wave value is well below the estimate from a serological survey (Portia Mutevedzi - MRC, personal communication). The estimated values of $\mathrm{R}_{0}$ ' for IFR=0.53 range from 2.3 to 2.6 for the cases under consideration (Supplementary Table 3.1), and are indicative of substantial increases (109-136\%) in transmissibility following the appearance of the B.1.351 variant. For values of IFR in the mid (IFR=0.68) and upper (IFR=0.82) globally reported ranges, the modelled accumulated infections by the end of the first and second waves are deemed to be unrealistically low in comparison to the surveyed seroprevalence, indicating that these values of the IFR would be overestimates for the Gauteng population (Supplementary Table 2.1).

The projected propagation of infections in the Gauteng province beyond 7 March 2021 is shown in Figure 3, assuming an IFR of 0.31 . In these simulations, it is assumed that $R_{0}$ ' is restricted to 1.4 between 7 March and 1 April 2021, which is sufficient to prevent the resurgence of infections during this time. From 2 April onwards, it is assumed that reduced adherence to the existing Level $1 \mathrm{NPI}$ in South Africa will increase Ro', according to two scenarios. The first is a worst case scenario, where $\mathrm{R}_{0}$ ' assumes the same value it had during the second wave of infections in Gauteng (Supplementary Table 3.1). The second is a best-case scenario, where $R_{0}$ ' remains restricted to a value of 1.6 (lower that any of the plausible $R_{0}$ ' values constructed for the second wave in Gauteng and two other provinces (Supplementary Tables 3.1 to 3.3; Methods). Under the worst case scenario, even for the case of persistent immunity, a third wave forms and peaks late in May 2021, with an amplitude similar to those of the first two waves (Figure 3a). A further $30 \%$ of the population is projected to be infected during the $3^{\text {rd }}$ wave, before community immunity is reached to sustain interruption in chains of transmission of the virus. For the cases where immunity is lost and reinfections occur, the third wave of infections can be substantially larger in amplitude than the first two waves (in the absence of strengthening NPI or widespread vaccination; Figures $3 \mathrm{~b}$ to $\mathrm{d}$ ). In these cases the third wave peaks earlier, in early May 2021. Fourth waves of infection also form for all these cases, peaking as early as November 2021 for the case of immunity lost within six months (Figure $3 d$ ). Restricting $R_{0}$ ' to 1.6 is sufficient to prevent the formation of a $3^{\text {rd }}$ wave for the case of persistent immunity (Figure 3a), and delays its formation and restricts its amplitude to about $50 \%$ or less of that of the second wave for all the scenarios of reinfection considered (Figure $3 \mathrm{~b}$ to $d$ ).

\section{Western Cape}

Reported cases (Figure 2a) and excess deaths (Figure 2e) suggest that the second wave peaked at 2 to 3 times the amplitude of the first in the Western Cape. This behavior can be replicated in the modelled infection curves by assuming an IFR of 0.31 (Figure 4, black lines). For this value of the IFR $26 \%$ of the population is simulated to have been infected by the end of August 2020 (after the first wave; Supplementary Table 2.2), which is somewhat lower than the 35-45\% seroprevalence recorded in selected communities in the Western Cape (Hsiao et al., 2020). The value of $R_{0}$ ' reconstructed for the first wave of infections is close to 1.1 (Supplementary Table 3.2). Accumulated infections are modelled to be about $75 \%$ of the population by 7 March 
(Supplementary Table 3.2), post the second wave. The second wave is associated with values of $\mathrm{R}_{0}$ ' ranging between 1.7 and 2.4 for the various cases under consideration (Supplementary Table 3.2), which represents increases in transmissibility of 54-100 \% compared to the first wave.

For an IFR of 0.2 , the modelling is indicative of $41 \%$ of infections by the end of the August 2021 (occurring in association with the first wave), and in the upper range of the fraction infections indicated for August 2020 by serological testing undertaken in selected communities (Hsiao et al., 2020). However, for the case of a persistent disease-induced immunity in an initially fullysusceptible population, this leaves an insufficient number of susceptible people for a secondwave of infections to reach three times the amplitude of the first, even with a high-force of infection. For the cases where immunity is lost, a second wave of observed amplitude can be generated, but this requires $115 \%$ of the population having been infected by 7 March 2021 (Supplementary Table 2.2). That is, more than $70 \%$ of the population is simulated to have been infected during the second wave. Values of the IFR substantially larger than 0.31 also produce reconstructed infection curves that are implausible. For an IFR of $0.53(0.82), 12 \%(10 \%)$ of the population is modelled to be infected by the end of August 2020, which substantially lower than the fraction of the population found to have been infected by August 2020 in the serological testing. By 7 March 2021, 34\% and $28 \%$ of the population are modelled to have been infected for levels of the IFR of 0.53 and 0.82 , respectively (Supplementary Table 2.2).

Assuming an IFR of 0.31 , the projected propagation of Covid-19 through 2021 is shown as a function of the various cases of immunity under consideration. Noteworthy is that even when assuming the values of $R_{0}$ ' reconstructed for the second wave (red lines), no further substantial growth in the infection curves are simulated for early 2021, suggesting that in all cases a form of community resistance has set in (contradicting the case of Gauteng Province). For the case of persistent immunity (Figure 4a), no third wave of infections form, given that community resistance has set in with about $75 \%$ of the population having been infected by 7 March 2021 . However, for the remaining three cases under consideration, third waves of infection form and peak between August and December 2021 (Figure $4 \mathrm{~b}$ to d). For the cases of immunity lost in twelve months, the third waves are about half the amplitude of the first, but of similar amplitude to the first for the case where immunity is lost in six months.

\section{Eastern Cape}

A second wave of infections first emerged in South Africa in the Eastern Cape in October 2020 (Figure 2b). The wave (using weekly excess deaths as measure, Figure 2f) peaked at about twice the amplitude of the first wave in December 2020, ahead of strengthened NPI in late December 2020 (Figure 1, Supplementary Table 1). Although the Eastern Cape and Western Cape provinces in South Africa have roughly equal population sizes (Methods), accumulated excess deaths in the Eastern Cape were almost twice that in Western Cape by the end of the first wave in August 2020 (9 582 deaths vs 5567 deaths). This ratio increased by 7 March 2020, post the second wave in both provinces (33 070 deaths vs 16041 deaths; Figure 2f). This suggests that one or both of the infection rate and the IFR was substantially higher in the Eastern than in the Western Cape.

An IFR of 0.31 , which provides a good fit for disease dynamics in both the Western Cape and Gauteng provinces, implies $47 \%$ of the population having been infected by the end of August 2020 in the Eastern Cape (Supplementary Table 2.3). With such a high fraction of the population infected by the end of the first wave, it is not possible for a second wave twice that size to have peaked by December 2020, even in the experiments where immunity is lost within 6 months and where $50 \%$ of the population with disease-induced immunity from the first wave had lost that 
immunity in the presence of the B.1.351 variant. Even with the reconstructed accumulated infections in the range $98-162 \%$ (Supplementary Table 2.3), the observed number of excess deaths by 7 March 2021 can't be reconstructed.

Assuming an IFR of 0.53 yields $28 \%$ of infections by the end of August 2020 (Supplementary Table 2.3), with Ro' for the first wave estimated to be 1.5 (Supplementary Table 3.3). This leaves a sufficient number of people susceptible for infection to produce a second wave of twice the amplitude of the first, in December 2020, with accumulated infections reaching about $94 \%$ by 7 March 2020. $R_{0}$ ' of the second wave is estimated to range between 1.8 and 2.8 (representing increases in transmissibility between the first and second wave of 20-87\%) (Supplementary Table 3.3). The medical infrastructure in the Eastern Cape has a lower capacity than the Western Cape and Gauteng provinces, consistent with the IFR being higher in the Eastern Cape.

At higher values of the IFR of 0.68 and 0.82 , the fractions of the population infected after the first wave are 22 and $18 \%$, respectively, values that are deemed to be at the lower end of plausibility (Supplementary Table 2.3). The corresponding fractions of accumulated infections by 7 March 2021 are about $73 \%$ and $61 \%$ (Supplementary Table 2.3). The reconstructed values of $R_{0}$ ' for IFR=0.68 range between 1.6 and 2 (Supplementary Table 3.3). In other words, the higher IFR and thus fewer infections inversed from excess deaths leave a large portion of the population susceptible, and thus do not require substantially larger values of $\mathrm{R}_{0}$ ' (compared to the first wave) for the scenarios of reinfection. This contradicts the notion of increased transmissibility in the Eastern Cape during the second wave, in the presence of the B.1.351 variant and reduced NPI.

Assuming an IFR of 0.53 , the projected propagation of Covid-19 in the Eastern Cape is shown in Figure 5. Given the high portion of accumulated infections by 7 March 2021, community resistance would prevent the formation of a third wave in the first half of 2021 for all cases under consideration. For the case where NPI is assumed to restrict transmission through to the end of 2021 (blue lines), a third wave of infections may not form in 2021, unless the duration of diseaseinduced immunity is short (six months), in which case a third wave of infections may form in the summer of 2021 (Figure 5d). If NPI is relaxed sooner, such a wave may form in the spring of 2021 , even if the duration of immunity is twelve months (Figure $5 b$ and c, red lines). In all these cases, the third wave is likely to be smaller in amplitude than the first, due to the smaller pool of susceptible people.

\section{Does cross-reactive immunity protect against infection?}

Under the assumptions of both wide-spread (30\%, Methods) pre-existing cross-immunity protecting against infection and disease-induced immunity being long lasting, second waves of the observed amplitude in the Eastern and Western Cape can't be replicated. However if it is assumed that immunity (including pre-existing cross-reactive immunity) is lost in periods of twelve or six months, or if a large faction of the immune population is not protected against the B.1.351 variant, sufficient pools of susceptible individuals become available for the model to replicate the observed number of excess deaths and associated waves of infection. In this latter scenario the effects of pre-existing cross-immunity diminishes over time and the disease dynamics approach that of a fully susceptible population. 


\section{Discussion}

Covid-19 propagation dynamics in South Africa are consistent with a fully susceptible population and IFR values of $0.31-0.53$, below or at the lower limit of international estimates of the IFR (Meyerowitz-Katz and Merone, 2020). It is possible that the relatively young population age structure in South Africa may have contributed to a higher proportion of mild or asymptomatic cases (Promislow, 2020) and relatively low IFR. On the other hand, South Africa has a high prevalence of comorbidity factors, including obesity, hypertension, diabetes, as well as tuberculosis and HIV, which were expected to increase fatal vulnerability to Covid-19 (Boulle et al., 2020; UNAIDS, 2020; TBFACTS, 2020; Rice et al., 2020). It is thus plausible that pre-existing cross-protection against severe manifestation of Covid-19 disease, possibly obtained from frequent exposure to common cold coronaviruses in South Africa's low-income and high density settlements, has played an important role in the manifestation of a low IFR in the country.

The modelling indicates that the first wave of infections plausibly left close to $30 \%$ of the population infected across the three provinces. Even if disease-induced immunity lasts for many years that left a sufficient pool of susceptible people for second waves of amplitude two to three times that of the first to develop. Such waves indeed occurred in the Eastern and Western Cape provinces, but in the Gauteng province the second wave had a similar amplitude to the first, possibly because NPI was strengthened nationally in time to head off the late-developing wave. Under assumptions of disease-induced immunity being lost within a year, or where immunity to earlier strains of SARS-CoV-2 conferred only partial immunity to the B.1.351 variant, even larger pools of susceptible people were available, from which the second wave of infections formed. In the Western and Eastern Cape Provinces of South Africa, transmissibility is estimated to have been be 20-100\% higher during the second wave of infections compared to the first, while in Gauteng province, transmissibility was $136-175 \%$ higher compared to the first wave. The enhanced transmissibility overall in South Africa argues for higher transmissibility of the B.1.351 variant, and the differences between provinces may be due to differences in NPI compliance (ahead of South Africa strengthening measures and more strongly enforced compliance late in December 2020).

South African Covid-19 propagation dynamics are most easily and plausibly explained by not assuming initial cross-immunity protecting against infection. The propagation dynamics provide no evidence that cross-immunity providing complete protection against infection was widespread, though cross-immunity may have provided some protection against infection by early Covid-19 variants. In particular, the magnitude of the second wave of infection in the Western and Eastern Cape provinces can't be replicated if wide-spread cross-immunity protecting against infection existed for the B.1.351 variant and diseased-induced immunity is long-lasting and effective across variants. On the other hand, if the duration of both pre-existing cross-reactive immunity and disease-induced immunity is only six to twelve months, the formation of high amplitude second waves of infection can be explained. In such a case, any effects of pre-existing cross-reactive immunity (against infection) on the disease dynamics quickly diminishes. This finding is important in the context of designing vaccination strategies in the country and more generally in low income countries.

The duration of disease-induced immunity in relation to the strength of NPI are key factors (other than the rate of vaccination and the evolution of further variants that are immune evasive) controlling the likelihood of the development of third or fourth waves of Covid-19 in South Africa in 2021. If immunity is effective across variants and lasts for many years, third waves are unlikely to occur in the Western and Eastern Cape provinces, given that the fraction of the population infected during the first two waves is close to the inferred herd immunity threshold. For immunity 
lasting six to twelve months, the potential exists in the Gauteng Province for a third wave of infections larger in magnitude than the first two waves, peaking during late autumn or the winter of 2021, and for a fourth wave to occur in the summer of 2021. Strengthened NPI and/or widespread vaccinations will be needed to prevent or dampen such a resurgence. In the Western and Eastern Cape provinces, where larger fractions of the population were infected during the first two waves, third waves of infection are likely to be smaller in amplitude than the earlier waves, and are projected to peak later than in Gauteng.

\section{References}

Boulle A, Davies M-A, Hussey H, et al. Risk factors for COVID-19 death in a population cohort study from the Western Cape Province, South Africa. Clin Infect Dis. 2020; doi:10.1093/cid/ciaa1198.

Bradshaw D, Laubscher R, Dorrington R, Groenewald P, Moultrie T. Report on weekly deaths in South Africa. 1 January - 13 October 2020 (Week 41). Burden of Disease Research Unit South African Medical Research Council. 20 October 2020. https://www.samrc.ac.za/reports/report-weekly-deaths-south-africa

Braun J, et al. SARS-CoV-2-reactive T cells in healthy donors and patients with COVID-19. Nature 2020; https://doi.org/10.1038/s41586-020-2598-9.

Britton T, Ball F, Trapman P. A mathematical model reveals the influence of population heterogeneity on herd immunity to SARS-CoV-2. Science 2020; 369: 846-849.

Fontanet A and Cauchemez S. COVID-19 herd immunity: where are we? Nature Reviews Immunology 2020; 20: 583-584. https://doi.org/10.1038/s41577-020-00451-5.

Garba SM, Lubuma JM and Tsanou B. Modeling the transmission dynamics of the covid-19 pandemic in South Africa. Mathematical Biosciences 2020; 108441.

https://doi.org/10.1016/j.mbs.2020.108441.

Greaney A.J., Loes A.N., Crawford K.H.D., Starr T.N., Malone K.D., Chu H.Y. and Bloom J.D. Comprehensive mapping of mutations in the SARS-CoV-2 receptor-binding domain that affect recognition by polyclonal human plasma antibodies. Cell Host and Microbe 2021; 29: 463-476.e6. https://doi.org/10.1016/i.chom.2021.02.003.

Grifoni, A. et al. Targets of T cell responses to SARS-CoV-2 coronavirus in humans with COVID19 disease and unexposed individuals. Cell 2020; 181: 1489-1501.

Hsiao M., et al. Covid-19 Special Public Health Surveillance Bulletin 2020; 18 (5). National Institute for Communicable Diseases.

Li R. et al. Substantial undocumented infection facilitates the rapid dissemination of novel coronavirus (SARS-CoV-2). Science 2020; 368: 489-493.

Lindestam Arlehamna CS, Sette A and Peters B. Lack of evidence for BCG vaccine protection from severe COVID-19. PNAS 2020; 117: 25203-25204. 
Mbow M, Lell B, Jochems SP, Cisse B, Mboup S, Dewals BG, Jaye A, Dieye A and Yazdanbakhsh M. COVID-19 in Africa: Dampening the storm? Science 2020; 369: 624-626. DOI: 10.1126/science.abd3902.

Meehan MT, et al. Modelling insights into the COVID-19 pandemic. Paediatric Respiratory Reviews 2020; 35: 64-69. https://doi.org/10.1016/i.prrv.2020.06.014.

Meyerowitz-Katz G. and Meronec L. A systematic review and meta-analysis of published research data on COVID-19 infection fatality rates. International Journal of Infectious Diseases 2020; 101 138-148.

Miller JC. A Note on the Derivation of Epidemic Final Sizes. Bull Math Biol 2012; 74: 2125-2141. DOI 10.1007/s11538-012-9749-6.

Moyo-Gwete et al. SARS-CoV-2 501Y.V2 (B.1.351) elicits cross-reactive neutralizing antibodies. bioRxiv preprint 2021; doi: https://doi.org/10.1101/2021.03.06.434193.

Nature (2020). Nature News in Focus. Q\&A. South Africa's top coronavirus adviser: 'Our epidemic could exceed a million cases'. Nature 2020; 583: 672. doi: https://doi.org/10.1038/d41586-02002216-5.

NICD (2020a). NICD Daily Report and Commentary, 08 October 2020. National Institute for Communicable Diseases. https://www.nicd.ac.za/diseases-a-z-index/covid-19/surveillancereports.

NICD (2020b). NICD COVID-19 Surveillance in Selected Hospitals. 07 January 2021. https://www.nicd.ac.za/wp-content/uploads/2021/01/Datcov19 National Export.07Jan.pdf

O'Neill LAJ and Netea MG. BCG- induced trained immunity: can it offer protection against COVID19? Nature 2020; 20: 336-337.

Our World in Data (2021). South Africa: Coronavirus Pandemic Country Profile. https://ourworldindata.org/coronavirus/country/south-africa.

Promislow DEL. A Geroscience Perspective on COVID-19 Mortality. J Gerontol A Biol Sci Med Sci 2020; 75: e30-e33. doi:10.1093/gerona/glaa09.

Rice BL, et al. High variation expected in the pace and burden of SARS-CoV-2 outbreaks across sub-Saharan Africa. Nature Medicine 2021; 27: 447-453. https://doi.org/10.1038/s41591-02101234-8.

ScienceMag (2020). A Kenyan health economist investigates the pandemic's puzzling course in his country. doi:10.1126/science.abg3420.

Sette A and Crotty S. Pre-existing immunity to SARS-CoV-2: the knowns and unknowns. Nature Reviews Immunology 2020; 20: 457-458.

Stiegler N and Bouchard JP. South Africa: challenges and successes of the COVID-19 lockdown. Annales Médico-Psychologiques 2020; 178: 695-698.

https://doi.org/10.1016/j.amp.2020.05.006 
TBFACTS (2020). https://tbfacts.org/tb-statistics-south-africal.

2

3

Uoga et al. Seroprevalence of anti-SARS-CoV-2 IgG antibodies in Kenyan blood donors. Science 2021; 371: 79-82.

UNAIDS (2020). https://www.unaids.org/en/regionscountries/countries/southafrica.

Volz et al. Transmission of SARS-CoV-2 Lineage B.1.1.7 in England: Insights from linking epidemiological and genetic data. MedRxiv 2020. doi: https://doi.org/10.1101/2020.12.30.20249034

Wassenaar TM, Buzard GS and Newman DJ. BCG vaccination early in life does not improve COVID-19 outcome of elderly populations, based on nationally reported data. Letters in Applied Microbiology 2020; doi:10.1111/lam.13365.

Wibmer, C.K., Ayres, F., Hermanus, T. et al. (2021). SARS-CoV-2 501Y.V2 escapes neutralization by South African COVID-19 donor plasma. Nat Med. https://doi.org/10.1038/s41591-021-01285-x 


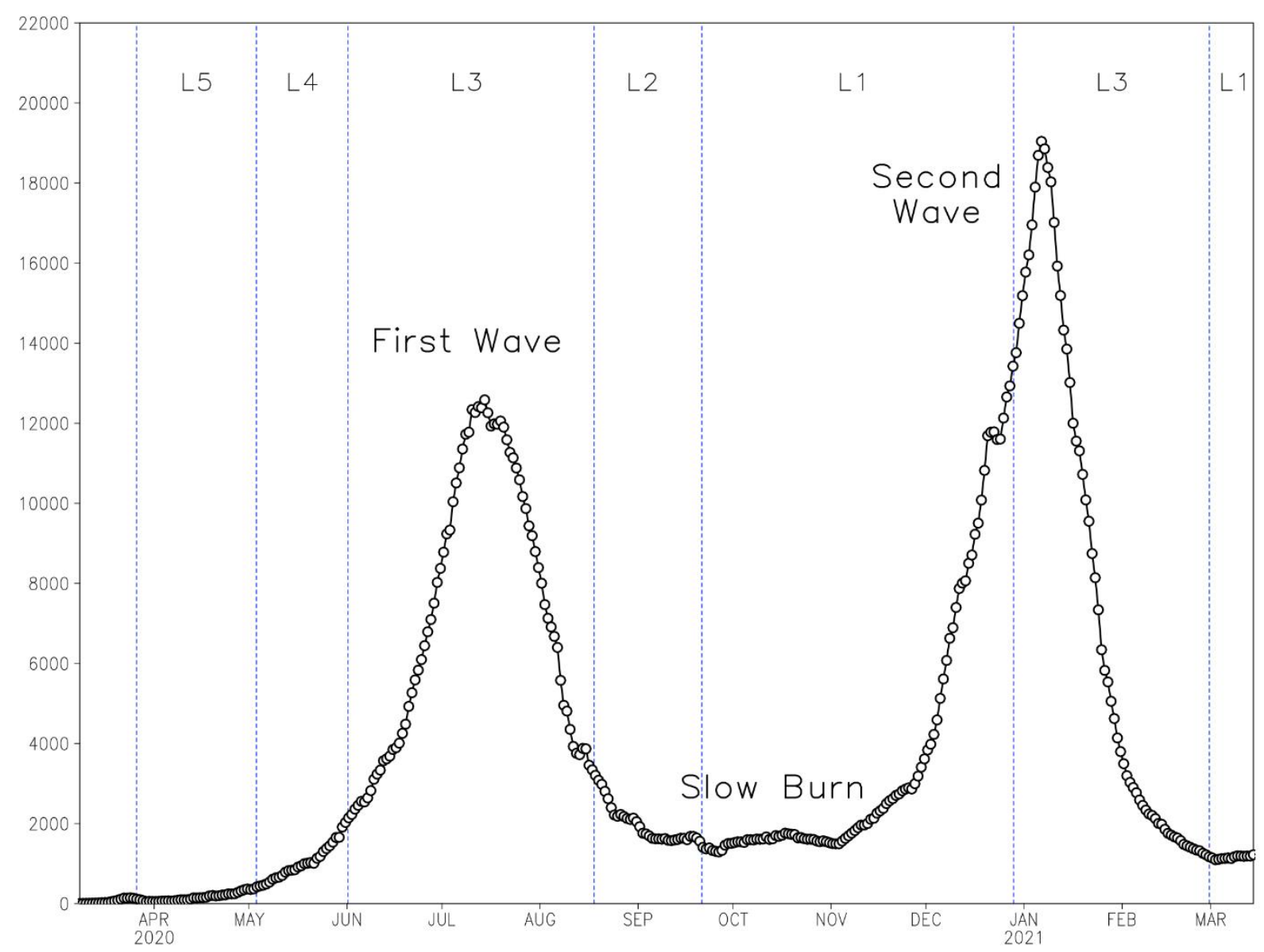

Figure 1: Propagation of Covid-19 in South Africa between 8 March 2020 and 14 March 2021, as described by the seven day centered moving average of daily reported cases. These numbers are substantially lower than actual cases due to limited testing. The 'levels' refer to NPI states, with 5 being the strictest. 
Western Cape infections

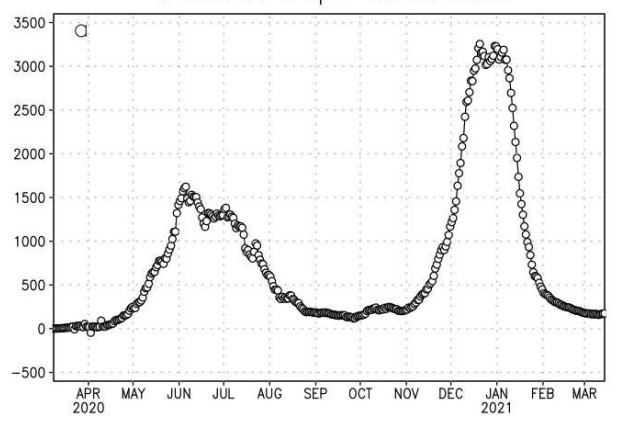

Eastern Cape infections

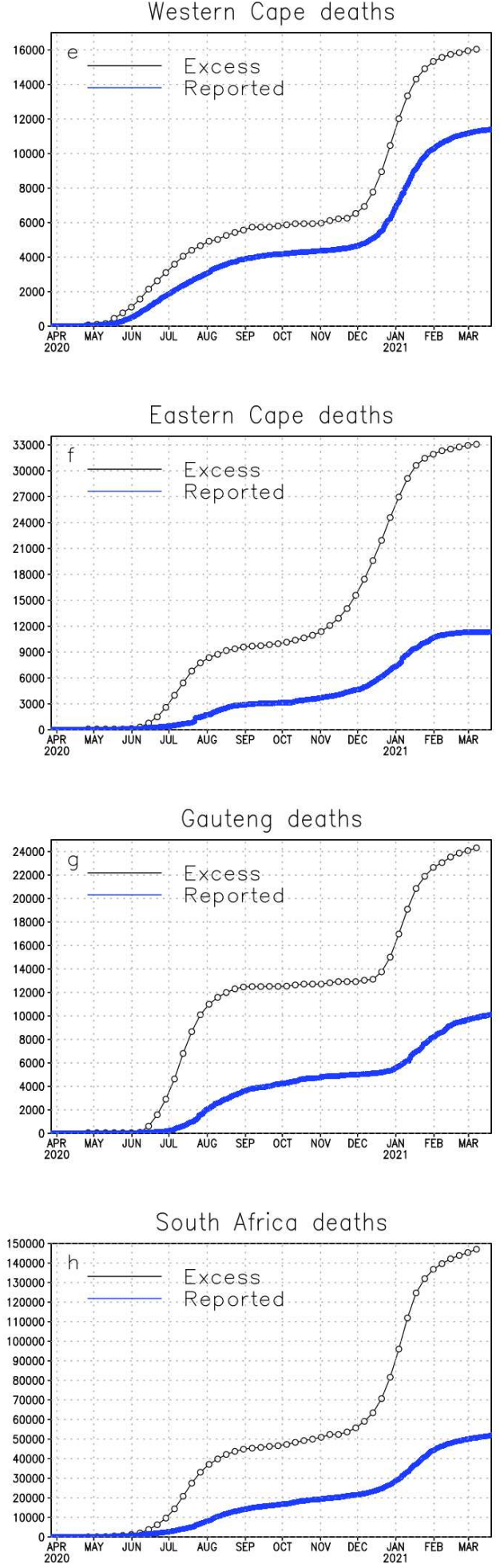

1

2

3

4

5 6

Figure 2: Left panels: Seven-day centered moving averages of daily reported Covid-19 infections for (a) Western Cape, (b) Eastern Cape, (c) Gauteng and (d) South Africa between 8 March 2020 and 14 March 2021. Right panels: Same as for the left panels, but showing accumulated excess (weekly, black lines) and reported (daily, blue lines) Covid-19 deaths through to 7 March and 19 March 2021, respectively. 

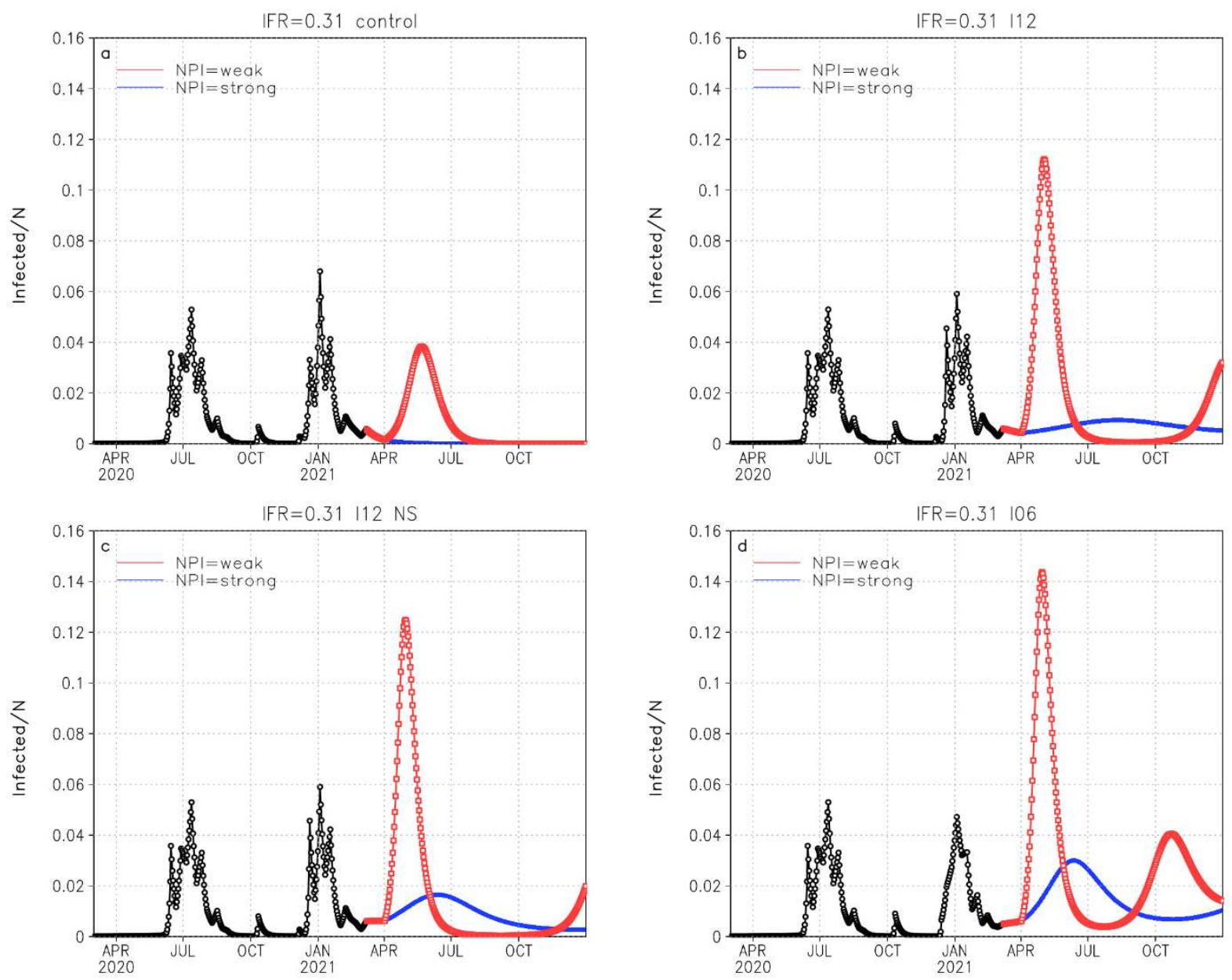

Figure 3: The reconstructed Covid-19 infections in the Gauteng province of South Africa for the period through to 7 March 2021 (black lines), and projected infections until the end of 2021 (blue and red lines), for an IFR of 0.31. Panel a represents the case where disease-induced immunity is ever lasting. Panel b represents immunity lasting for 12 months. Panel $c$ represents the case where immunity lasts for 12 months, but where the arrival of the new B.1.351 variant in late November 2020 was associated with $50 \%$ of the immune population at the time becoming susceptible to the new variant. Panel $d$ is similar to panel $b$, but with immunity lost after six months. For the projections it is assumed that NPI will restrict $R_{0}$ ' to 1.4 through to 1 April 2021. The red lines represent a worst case scenario where $\mathrm{R}_{0}$ ' assumes the value as reconstructed for the second wave (Supplementary Table 3.1) from 2 April 2021 onwards. The blue lines represent a best-case scenario where NPI restricts Ro' to 1.6 from 2 April onwards. 



Figure 4: The reconstructed Covid-19 infections in the Western Cape province of South Africa for the period through to 7 March 2021 (black lines), and projected infections until the end of 2021 (blue and red lines), for an IFR of 0.31. Panel a represents the case where disease-induced immunity is ever lasting. Panel b represents immunity lasting for 12 months. Panel c represents the case where immunity lasts for 12 months, but where the arrival of the new B.1.351 variant in early November 2020 was associated with $50 \%$ of the immune population at the time becoming susceptible to the new variant. Panel $d$ is similar to panel $b$, but with immunity lost after six months. For the projections it is assumed that NPI will restrict $R_{0}$ ' to 1.4 through to 1 April 2021. The red lines represent a worst case scenario where $\mathrm{R}_{0}$ ' assumes the value as reconstructed for the second wave (Supplementary Table 3.1) from 2 April 2021 onwards. The blue lines represent a best-case scenario where NPI restricts $R_{0}$ ' to 1.6 from 2 April onwards. 



Figure 5: The reconstructed Covid-19 infections in the Eastern Cape province of South Africa for the period through to 7 March 2021 (black lines), and projected infections until the end of 2021 (blue and red lines), for an IFR of 0.53. Panel a represents the case where disease-induced immunity is ever lasting. Panel $b$ represents immunity lasting for 12 months. Panel c represents the case where immunity lasts for 12 months, but where the arrival of the new B.1.351 variant in early October 2020 was associated with $50 \%$ of the immune population at the time becoming susceptible to the new variant. Panel $d$ is similar to panel $b$, but with immunity lost after six months. For the projections it is assumed that NPI will restrict $R_{0}$ ' to 1.4 through to 1 April 2021. The red lines represent a worst case scenario where $\mathrm{R}_{0}$ ' assumes the value as reconstructed for the second wave (Supplementary Table 3.1) from 2 April 2021 onwards. The blue lines represent a best-case scenario where NPI restricts $\mathrm{R}_{0}$ ' to 1.6 from 2 April onwards. 


\section{Methods}

Limitations in testing for COVID-19, with approximately 80 tests per 1000 population having been undertaken in South Africa by Oct 2020 (Our world in data), inadvertently results in the reported numbers of COVID-19 being an underestimate of the actual number of infections, as is the case in most other countries (Li et al., 2020). The paper therefore makes use of this measure only as a qualitative measure of the progression of the disease in South Africa and its provinces, but it is not used quantitatively in the inverse modelling that is performed. Deaths directly attributable to Covid-19 (UP, 2020) are also an underestimate of the actual number, due to the limited availability of polymerase chain reaction (PCR) confirmations. However, deaths from whatever cause must be reported to the authorities, and the reporting rate to the vital registration system is high: victims need to be buried and death benefits are to be claimed. The study thus relies on estimated weekly 'excess deaths', of which Covid-19 attributable deaths make up the major component in South Africa (Bradshaw et al., 2020), as the key measure of disease propagation dynamics. Data on weekly excess deaths were available through to the week ending 7 March 2021 at the time of composing this paper. The fraction of reported Covid-19 deaths to excess deaths in South Africa is highly variable between provinces, from $34 \%$ in the Eastern Cape, $41 \%$ in Gauteng to $70 \%$ in the Western Cape by 7 March 2021. Reported cases, reported deaths and excess deaths are displayed in Figure 2 for the Western and Eastern Cape and Gauteng provinces, and for South Africa, for the period March 2020 to March 2021.

The study relies on serological surveys undertaken in the Western Cape (Hsiao et al., 2020) and Gauteng (Portia Mutevedzi - MRC, personal communication), which suggested that by the end of the first wave in August 2020, about 35-45\% of people in the communities surveyed in the Western Cape had been infected, and about $25 \%$ in Gauteng. These numbers are used to verify the model simulations in terms of their ability to represent the fraction of the various populations infected by the end of the first wave.

We applied a Susceptible-Infected-Recovered-Dead (SIRD) model separately to the Eastern Cape, Western Cape and Gauteng provinces of South Africa:

$$
\begin{gathered}
\frac{d S}{d t}=-\frac{\alpha}{N} S I+\omega R ; \\
\frac{d I}{d t}=\frac{\alpha}{N} S I-\beta I-\gamma I ;
\end{gathered}
$$

$$
\frac{d R}{d t}=\beta I-\omega R ;
$$

$$
\frac{d D}{d t}=\gamma I .
$$

Each province is assumed to have an isolated, homogeneous and initially fully susceptible population of size N (6.7 million for the Eastern Cape, 7 million for the Western Cape and 15.5 million for Gauteng). S, I, R and D are the number of susceptible, infected, recovered and dead individuals respectively, at a given moment in time, and they sum to the total population, $\mathrm{N}$ (assumed to remain constant). The daily recovery rate $\beta=0.16 d^{-1}$ is constant in all the simulations performed and consistent with estimates used in other studies (Anastassopoulou et 
al., 2020; Baker et al., 2020). The daily infection rate is $\alpha$ and the mortality rate is $\gamma$. Note that the IFR $=\gamma / \beta$ and $R_{0}=\alpha /(\gamma+\beta)$. The rate at which immunity is lost is $\omega$.

The model is initialized with $S_{t=0}=\left(1-c_{r}\right) N-I_{t=0}$ and $R_{t=0}=c_{r} N$, where $\mathrm{c}_{\mathrm{r}}$ is the fraction of the population with pre-existing cross-immunity against infection. $I_{t=0}=1$ and $D_{t=0}=0$. Given the substantial variations in the strength of NPI during the simulation period we do not attempt to identify the potential impacts of seasonality on infection rates (Baker et al., 2020; Engelbrecht and Scholes, 2021; Smit et al., 2020). The model also assumes that the IFR remains constant across the duration of the disease's propagation, an assumption that may not be valid given the emergence of the B.1.351 variant in November 2020.

The inverse application of the model reconstructs the modified reproduction number $R_{0}{ }^{\prime}=$ $\alpha_{m} /(\gamma+\beta)$ (with $\alpha_{m}$ the actual time-varying infection rate) for each value of $c_{r}$ and the IFR, and for the prescribed period that disease-induced immunity lasts, from the time-series of excess deaths. The model inversion distinguishes between the period up and until 29 April 2020 (from the onset of Covid-19 in South Africa to the end of stringent lockdown, Supplementary Table 1) and the subsequent period during which lockdown measures were progressively relaxed, albeit with ongoing advocacy for use of NPIs, and for which data on excess deaths are available. The model was initialized on the $5^{\text {th }}$ of March 2020 and integrated forward to 29 April 2020, the end of stringent lockdown. Model inversion solutions were obtained by systematically integrating the model for different values of $R_{0}$ ', in intervals of 0.0001 , and selecting the smallest value of $R_{0}$ ' that yields accumulated deaths equal to or greater than the recorded excess deaths at the end of this period. This process was then repeated for the period 6 May 2020 to 7 March 2021, with $R_{0}{ }^{\prime}$ reconstructed to match the weekly recorded excess deaths, always initializing from the latest state obtained for S, I, R and D.

Four scenarios of the duration of disease-induced immunity are explored in the simulations: immunity that lasts indefinitely, for twelve months, for six months, and immunity that generally lasts for twelve months but where $50 \%$ of the population in whom immunity was induced by prototype SARS-CoV-2 remained susceptible to the B.1.351 variant. Based on the dates for which the weekly numbers of excess deaths started to increase after the period of 'slow burn' that followed the first wave (Figure 1), the emergence date of the new B.1.351 variant was taken to be 7 October 2021 in the Eastern Cape, 8 November 2021 in the Western Cape and 30 November 2021 in Gauteng. For each of these four cases, a set of time-series of the modified reproduction number $\left(\mathrm{R}_{0}\right.$ ', representing the time-varying effects of NPI on $\mathrm{R}_{0}$ ) was reconstructed as a function of the IFR. In other words, the IFR is kept constant in each simulation, but different realisations of the IFR in its plausible range are explored. A recent review suggested $0.53 \%$ to $0.82 \%$ as the plausible range of the IFR (Meyerowitz-Katz and Merone, 2020), although other studies suggest values as low as 0.3 and as high as 1.2 (Fontanet and Cauchemez, 2020). Note that the effective reproduction number is related to $\mathrm{R}_{0}{ }^{\prime}$ by $R_{t},=S R_{0}{ }^{\prime} / N$. Values of $\mathrm{R}_{0}{ }^{\prime}$ are averaged over the sixweek period preceding the peak of the first wave, and the six week period preceding the peak of the second wave, for each of the provinces (Supplementary Tabled 3.1 to 3.3). To these values are referred to as the 'first wave' and 'second wave' values of $\mathrm{R}_{0}$ '. Accumulated infections, which may include reinfections, are tabled for the dates 30 August (post first-wave) and 7 March (post second-wave) for each of the provinces, in Supplementary Tables 2.1 to 2.3.

After 7 March 2021, the model is released from its 'spun-up' state to project forward to December 2021. It is assumed that continued adherence to NPI recommendations will restrict $R_{0}$ ' to 1.4 until 1 April 2021. A worst case and best case scenario are considered for the period 2 April 2021 onwards. For each of the cases of reinfection, the worst case scenario assumes a value of $\mathrm{R}_{0}$ ' as 
estimated from the second wave of infections (Supplementary Tables 3.1 to 3.3), whilst the best case scenario assumes a value of 1.6.

Note that in order to explore the potential effects of pre-existing cross-reactive immunity that protects against SARS-CoV-2 infection, the simulations described above are performed for either a fully susceptible population $\left(c_{r}=0\right)$ or with a fraction of the population $\left(c_{r}=0.3\right)$ having preexisting immunity protecting against infection.

The simulations do not take into account any pharmaceutical control measures (ie vaccination) that may become increasingly available in South Africa after April 2021. They thus effectively provide guidance on how the disease may evolve in South Africa in the absence of an efficient roll-out of Covid-19 immunization.

\section{References}

Anastassopoulou C, Russo L, Tsakris A and Siettos C. Data-based analysis, modelling and forecasting of the COVID-19 outbreak. PlosOne 2020; 1010.

DOI:10.1371/journal.pone.0230405.

Baker RE, Yang W, Vecchi GA, Metcalf CJE and Grenfell BT. Susceptible supply limits the role of climate in the early SARS-CoV-2 pandemic. Science 2020; DOI: 10.1126/science.abc2535.

Bradshaw D, Laubscher R, Dorrington R, Groenewald P, Moultrie T. Report on weekly deaths in South Africa. 1 January - 13 October 2020 (Week 41). Burden of Disease Research Unit South African Medical Research Council. 20 October 2020.

https://www.samrc.ac.za/reports/report-weekly-deaths-south-africa

Engelbrecht F.A. and Scholes R. J. (2021). Test for Covid-19 seasonality and the risk of second waves. One Health 12100202.

Fontanet A and Cauchemez S. COVID-19 herd immunity: where are we? Nature Reviews Immunology 2020; 20: 583-584. https://doi.org/10.1038/s41577-020-00451-5.

Hsiao M., et al. Covid-19 Special Public Health Surveillance Bulletin 2020; 18 (5). National Institute for Communicable Diseases.

Li R. et al. Substantial undocumented infection facilitates the rapid dissemination of novel coronavirus (SARS-CoV-2). Science 2020; 368: 489-493.

Meyerowitz-Katz G. and Meronec L. A systematic review and meta-analysis of published research data on COVID-19 infection fatality rates. International Journal of Infectious Diseases 2020; 101 138-148.

Our World in Data (2021). South Africa: Coronavirus Pandemic Country Profile. https://ourworldindata.org/coronavirus/country/south-africa.

Smit AJ, Fitchett JM, Engelbrecht FA, Scholes RJ, Dzhivhuho G and Sweijd NA. Winter is Coming: A southern Hemisphere Perspective of the Environmental Drivers of SARS-CoV-2 and the Potential Seasonality of COVID-19. Int. J. Environ. Res. Public Health 2020; 17: 5634. doi:10.3390/ijerph17165634. 
UP (2020). COVID 19 ZA South Africa Dashboard. Data Science for Social Impact Research Group, University of Pretoria. https://datastudio.google.com/reporting/1b60bdc7-bec7-44c9ba29-be0e043d8534/page/hrUIB.

4

4

5

6

7

8

9

10

11

12

13

14

15

16

17

18

19

20

21

22

23

24

25

26

27

28

29

30

31

32

33

34

35

36

37

38

39

40

41

42

43

44

45

46

47

48

49

50

51 


\section{Supplementary Information}

Supplementary Table 1: The non-pharmaceutical interventions aimed at reducing the peak of Covid-19 infections and deaths in South Africa were implemented in discrete stages. The degree to which the population conformed with the restrictions - particularly those related to maskwearing and social distancing - was highly variable, but generally very far below complete, especially after about June 2020.

\begin{tabular}{|c|c|c|}
\hline From & To & Regulations \\
\hline $\begin{array}{l}5 \\
\text { Mar } \\
2020\end{array}$ & $\begin{array}{l}25 \text { Mar } \\
2020\end{array}$ & Cessation of international travel other than repatriation missions. \\
\hline $\begin{array}{l}26 \\
\text { Mar } \\
2020\end{array}$ & $\begin{array}{l}\text { 3 May } \\
2020\end{array}$ & $\begin{array}{l}\text { Level } 5 \text { lockdown. Shelter-in-place for entire population, without visitors, } \\
\text { leaving only to buy food or for medical treatment. Only essential workers } \\
\text { at work (police, military, utilities, food provision, health workers). Alcohol } \\
\text { and tobacco ban, all drinking and eating establishments closed, no } \\
\text { public transport, international borders closed, travel between provinces } \\
\text { by permit only. }\end{array}$ \\
\hline $\begin{array}{l}4 \\
\text { May } \\
2020\end{array}$ & $\begin{array}{l}31 \text { May } \\
2020\end{array}$ & $\begin{array}{l}\text { Level 4. About } 1 / 3^{\text {rd }} \text { of employed return to work (all agriculture, financial } \\
\text { services, IT services; } 50 \% \text { of mining) with hygiene and distancing } \\
\text { protocols. Open-air exercise permitted within } 5 \mathrm{~km} \text { of home, no gyms. } \\
\text { Masks compulsory in public. }\end{array}$ \\
\hline $\begin{array}{l}1 \\
\text { June } \\
2020\end{array}$ & $\begin{array}{l}\text { 17 Aug } \\
2020\end{array}$ & $\begin{array}{l}\text { Level 3. Partial school re-opening (mostly final year grades, learners } \\
\text { returning alternate days, most learning online, no school sports). E- } \\
\text { commerce permitted, clothing stores open, take-away foods permitted, } \\
\text { government service offices open. Automobile, cement, steel industries, } \\
\text { road maintenance, railways open. Public transport open all hours, with } \\
\text { distancing. }\end{array}$ \\
\hline $\begin{array}{l}18 \\
\text { Aug } \\
2020\end{array}$ & $\begin{array}{l}20 \text { Sep } \\
2020\end{array}$ & $\begin{array}{l}\text { Level 2. All retail stores open, including hairdressers. Family visits } \\
\text { allowed, limit of } 50 \text { people for gatherings (including funerals and } \\
\text { religious), parks open, curfew } 10 \text { pm to } 4 \text { am, drinking and eating } \\
\text { establishments open with spacing restrictions. Interprovincial travel for } \\
\text { any purpose permitted. }\end{array}$ \\
\hline $\begin{array}{l}21 \\
\text { Sep } \\
2020\end{array}$ & $\begin{array}{l}28 \text { Dec } \\
2020\end{array}$ & $\begin{array}{l}\text { Level 1. All sectors open, with distancing and hygiene protocols. No } \\
\text { sports events with spectators, conferences remain virtual, indoor } \\
\text { gatherings limited to } 250 \text { people. International travel from approved } \\
\text { destinations permitted. Almost all higher education facilities continue } \\
\text { predominantly teaching online, with limited physical presence. }\end{array}$ \\
\hline $\begin{array}{l}29 \\
\text { Dec } \\
2020\end{array}$ & $\begin{array}{l}28 \\
\text { February } \\
2021\end{array}$ & Level 3. \\
\hline $\begin{array}{l}1 \\
\text { Mar } \\
2021\end{array}$ & $\begin{array}{l}\text { Ongoing } \\
\text { by } 3 \text { April } \\
2021\end{array}$ & Level 1. \\
\hline
\end{tabular}


Supplementary Table 2.1: Accumulated infections in a fully susceptible population (expressed as a percentage of the total population $\mathrm{N}$ ) reconstructed by model inversion of excess mortality in the Gauteng province for the period post the first wave and post the second wave, as a function of the IFR, the duration of disease-induced immunity $(\mathrm{L})$ and fraction of the population infected during the first wave that retained that immunity against the B.1.351 variant $(\mathrm{N} \%)$.

\begin{tabular}{|c|c|c|c|c|}
\hline \multirow[t]{2}{*}{ IFR } & \multirow[t]{2}{*}{ (months) } & \multirow[t]{2}{*}{$\begin{array}{l}\mathbf{N} \\
(\%)\end{array}$} & \multicolumn{2}{|c|}{$\begin{array}{l}\text { Accumulated infections expressed as a percentage of } \\
\text { the total population } \mathrm{N}\end{array}$} \\
\hline & & & $\begin{array}{l}\text { Post first wave (30 } \\
\text { August 2020) }\end{array}$ & $\begin{array}{l}\text { Post second wave (7 } \\
\text { March 2021) }\end{array}$ \\
\hline 0.2 & $\infty$ & 100 & 41 & 79 \\
\hline 0.2 & 12 & 100 & 41 & 80 \\
\hline 0.2 & 12 & 50 & 41 & 80 \\
\hline 0.2 & 6 & 100 & 41 & 79 \\
\hline 0.31 & $\infty$ & 100 & 26 & 51 \\
\hline 0.31 & 12 & 100 & 26 & 51 \\
\hline 0.31 & 12 & 50 & 26 & 51 \\
\hline 0.31 & 6 & 100 & 26 & 51 \\
\hline 0.53 & $\infty$ & 100 & 15 & 30 \\
\hline 0.53 & 12 & 100 & 15 & 30 \\
\hline 0.53 & 12 & 50 & 15 & 30 \\
\hline 0.53 & 6 & 100 & 15 & 30 \\
\hline 0.68 & $\infty$ & 100 & 12 & 23 \\
\hline 0.68 & 12 & 100 & 12 & 23 \\
\hline 0.68 & 12 & 50 & 12 & 24 \\
\hline 0.68 & 6 & 100 & 12 & 23 \\
\hline 0.82 & $\infty$ & 100 & 10 & 20 \\
\hline 0.82 & 12 & 100 & 10 & 20 \\
\hline 0.82 & 12 & 50 & 10 & 20 \\
\hline 0.82 & 6 & 100 & 10 & 20 \\
\hline
\end{tabular}


Supplementary Table 2.2: Accumulated infections in a fully susceptible population (expressed as a percentage of the total population $\mathrm{N}$ ) reconstructed by model inversion of excess mortality in the Western Cape province for the period post the first wave and post the second wave, as a function of the IFR, the duration of disease-induced immunity $(\mathrm{L})$ and fraction of the population infected during the first wave that retained that immunity against the B.1.351 variant $(\mathrm{N} \%)$.

\begin{tabular}{|c|c|c|c|c|}
\hline IFR & \multirow{2}{*}{$\begin{array}{l}\text { (months) } \\
\end{array}$} & \multirow{2}{*}{$\begin{array}{l}\mathbf{N} \\
\end{array}$} & & $\begin{array}{l}\text { Accumulated infections expressed as a percentage of } \\
\text { the total population N }\end{array}$ \\
\cline { 5 - 6 } & $\begin{array}{c}\text { Post first wave (30 } \\
\text { August 2020) }\end{array}$ & $\begin{array}{c}\text { Post second wave (7 } \\
\text { March 2021) }\end{array}$ \\
\hline 0.2 & $\infty$ & 100 & 41 & 98 \\
\hline 0.2 & 12 & 100 & 41 & 115 \\
\hline 0.2 & 12 & 50 & 41 & 115 \\
\hline 0.2 & 6 & 100 & 41 & 115 \\
\hline 0.31 & $\infty$ & 100 & 26 & 74 \\
\hline 0.31 & 12 & 100 & 26 & 74 \\
\hline 0.31 & 12 & 50 & 26 & 75 \\
\hline 0.31 & 6 & 100 & 26 & 75 \\
\hline 0.53 & $\infty$ & 100 & 16 & 44 \\
\hline 0.53 & 12 & 100 & 16 & 44 \\
\hline 0.53 & 12 & 50 & 16 & 44 \\
\hline 0.53 & 6 & 100 & 16 & 44 \\
\hline 0.68 & $\infty$ & 100 & 12 & 34 \\
\hline 0.68 & 12 & 100 & 12 & 34 \\
\hline 0.68 & 12 & 50 & 12 & 34 \\
\hline 0.68 & 6 & 100 & 12 & 34 \\
\hline 0.82 & $\infty$ & 100 & 10 & 28 \\
\hline 0.82 & 12 & 100 & 10 & 28 \\
\hline 0.82 & 12 & 50 & 10 & 28 \\
\hline 0.82 & 6 & 100 & 10 & \\
\hline & & & & 28 \\
\hline
\end{tabular}


Supplementary Table 2.3: Accumulated infections in a fully susceptible population (expressed as a percentage of the total population $\mathrm{N}$ ) reconstructed by model inversion of excess mortality in the Eastern Cape province for the period post the first wave and post the second wave, as a function of the IFR, the duration of disease-induced immunity (L) and fraction of the population infected during the first wave that retained that immunity against the B.1.351 variant $(\mathrm{N} \%)$.

\begin{tabular}{|c|c|c|c|c|}
\hline IFR & \multirow{2}{*}{$\begin{array}{l}\mathbf{L} \text { (months) } \\
\end{array}$} & \multirow{2}{*}{$\begin{array}{l}\mathbf{N}) \\
\end{array}$} & & $\begin{array}{l}\text { Accumulated infections expressed as a percentage of } \\
\text { the total population N }\end{array}$ \\
\cline { 5 - 6 } & $\begin{array}{c}\text { Post first wave (30 } \\
\text { August 2020) }\end{array}$ & $\begin{array}{c}\text { Post second wave (7 } \\
\text { March 2021) }\end{array}$ \\
\hline 0.2 & $\infty$ & 100 & 73 & 96 \\
\hline 0.2 & 12 & 100 & 73 & 140 \\
\hline 0.2 & 12 & 50 & 73 & 161 \\
\hline 0.2 & 6 & 100 & 73 & 182 \\
\hline 0.31 & $\infty$ & 100 & 47 & 98 \\
\hline 0.31 & 12 & 100 & 47 & 127 \\
\hline 0.31 & 12 & 50 & 47 & 142 \\
\hline 0.31 & 6 & 100 & 47 & 162 \\
\hline 0.53 & $\infty$ & 100 & 28 & 93 \\
\hline 0.53 & 12 & 100 & 28 & 94 \\
\hline 0.53 & 12 & 50 & 28 & 94 \\
\hline 0.53 & 6 & 100 & 28 & 94 \\
\hline 0.68 & $\infty$ & 100 & 22 & 73 \\
\hline 0.68 & 12 & 100 & 22 & 73 \\
\hline 0.68 & 12 & 50 & 22 & 73 \\
\hline 0.68 & 6 & 100 & 22 & 61 \\
\hline 0.82 & $\infty$ & 100 & 18 & 61 \\
\hline 0.82 & 12 & 100 & 18 & 61 \\
\hline 0.82 & 12 & 50 & 18 & 61 \\
\hline 0.82 & 6 & 100 & 18 & \\
\hline & & & & 73 \\
\hline
\end{tabular}


7

Supplementary Table 3.1: $R_{0}$ ' reconstructed by model inversion of excess mortality in the Gauteng province for the first and second waves assuming a fully susceptible population, and as a function of the IFR, the duration of disease-induced immunity (L) and fraction of the population infected during the first wave that retained that immunity against the B.1.351 variant $(\mathrm{N})$.

\begin{tabular}{|c|c|c|c|c|}
\hline \multirow[t]{2}{*}{ IFR } & \multirow[t]{2}{*}{$\mathbf{L}$} & \multirow[t]{2}{*}{$\begin{array}{l}\mathbf{N} \\
(\%)\end{array}$} & \multicolumn{2}{|c|}{$\begin{array}{l}\text { Averages of } R_{0} \text { ' as estimated from the inversion of weekly } \\
\text { deaths }\end{array}$} \\
\hline & & & $\begin{array}{l}\text { First Wave peak (15 Jun to } \\
26 \text { July) }\end{array}$ & $\begin{array}{l}\text { Second wave peak ( } 30 \\
\text { November to } 10 \text { January) }\end{array}$ \\
\hline 0.31 & $\infty$ & 100 & 1.2 & 3.3 \\
\hline 0.31 & 12 & 100 & 1.1 & 2.9 \\
\hline 0.31 & 12 & 50 & 1.1 & 2.6 \\
\hline 0.31 & 06 & 100 & 1.1 & 2.8 \\
\hline 0.53 & $\infty$ & 100 & 1.1 & 2.6 \\
\hline 0.53 & 12 & 100 & 1.1 & 2.6 \\
\hline 0.53 & 12 & 50 & 1.1 & 2.5 \\
\hline 0.53 & 06 & 100 & 1.1 & 2.3 \\
\hline
\end{tabular}

Supplementary Table 3.2: $R_{0}$ ' reconstructed by model inversion of excess mortality in the Western Cape province for the first and second waves assuming a fully susceptible population, and as a function of the IFR, the duration of disease-induced immunity $(L)$ and fraction of the population infected during the first wave that retained that immunity against the B.1.351 variant (N).

\begin{tabular}{|l|l|l|c|c|}
\hline IFR & $\mathbf{L}$ & $\begin{array}{l}\mathbf{N} \\
\mathbf{( \% )}\end{array}$ & & $\begin{array}{l}\text { Averages of } \mathbf{R}_{\mathbf{0}} \text { ' as estimated from the inversion of weekly } \\
\text { deaths }\end{array}$ \\
\cline { 4 - 5 } & & $\begin{array}{c}\text { First Wave peak (25 May to } \\
\text { 5 July) }\end{array}$ & $\begin{array}{c}\text { Second wave peak (23 } \\
\text { November to 3 January) }\end{array}$ \\
\hline 0.31 & $\infty$ & 100 & 1.2 & 2.4 \\
\hline 0.31 & 12 & 100 & 1.1 & 1.8 \\
\hline 0.31 & 12 & 50 & 1.1 & 1.7 \\
\hline 0.31 & 06 & 100 & 1.1 & 1.9 \\
\hline 0.53 & $\infty$ & 100 & 1.1 & 1.8 \\
\hline 0.53 & 12 & 100 & 1.1 & 1.5 \\
\hline 0.53 & 12 & 50 & 1.1 & 1.5 \\
\hline 0.53 & 06 & 100 & 1.1 & 1.6 \\
\hline
\end{tabular}


1

\begin{tabular}{|l|l|l|l|c|}
\hline IFR & $\mathbf{L}$ & $\begin{array}{l}\mathbf{N} \\
\mathbf{( \% )}\end{array}$ & & \multicolumn{2}{|c|}{$\begin{array}{l}\text { Averages of } \mathbf{R}_{\mathbf{0}} \text { ' as estimated from the inversion of weekly } \\
\text { deaths }\end{array}$} \\
\cline { 4 - 6 } & & $\begin{array}{c}\text { First Wave peak (1 June to } \\
\text { 12 July) }\end{array}$ & $\begin{array}{c}\text { Second wave peak (16 } \\
\text { November to 27 December) }\end{array}$ \\
\hline 0.53 & $\infty$ & 100 & 1.5 & 2.8 \\
\hline 0.53 & 12 & 100 & 1.5 & 2.2 \\
\hline 0.53 & 12 & 50 & 1.5 & 1.8 \\
\hline 0.53 & 06 & 100 & 1.5 & 1.9 \\
\hline 0.68 & $\infty$ & 100 & 1.5 & 2.0 \\
\hline 0.68 & 12 & 100 & 1.5 & 1.8 \\
\hline 0.68 & 12 & 50 & 1.5 & 1.6 \\
\hline 0.68 & 06 & 100 & 1.5 & 1.6 \\
\hline
\end{tabular}

Supplementary Table 3.3: $\mathrm{R}_{0}$ ' reconstructed by model inversion of excess mortality in the Eastern Cape province for the first and second waves assuming a fully susceptible population, and as a function of the IFR, the duration of disease-induced immunity (L) and fraction of the population infected during the first wave that retained that immunity against the B.1.351 variant $(\mathrm{N})$. 
Figures

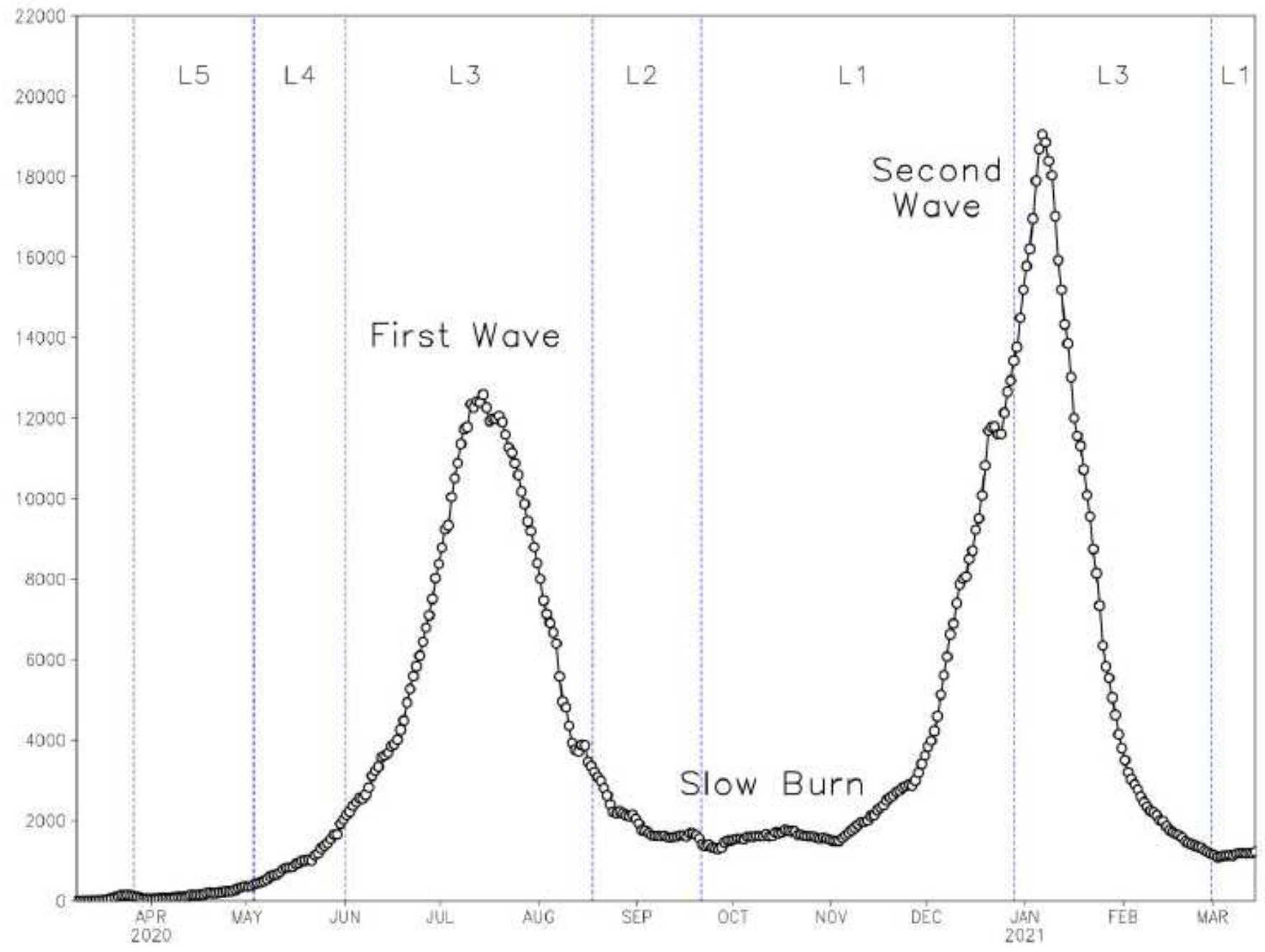

Figure 1

Propagation of Covid-19 in South Africa between 8 March 2020 and 14 March 2021, as described by the seven day centered moving average of daily reported cases. These numbers are substantially lower than actual cases due to limited testing. The 'levels' refer to NPI states, with 5 being the strictest. 
Western Cape infections

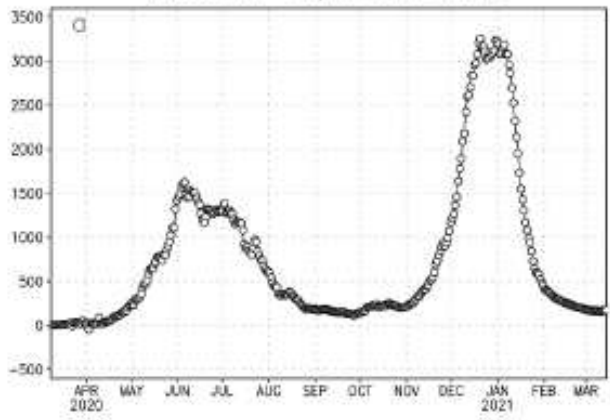

Eostern Cape infections

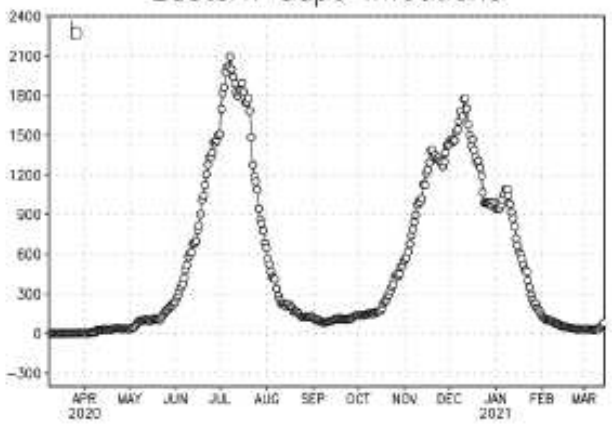

Gouteng infections

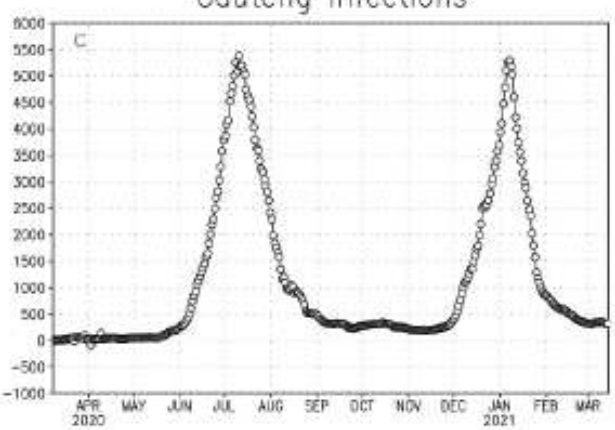

South Africa infections

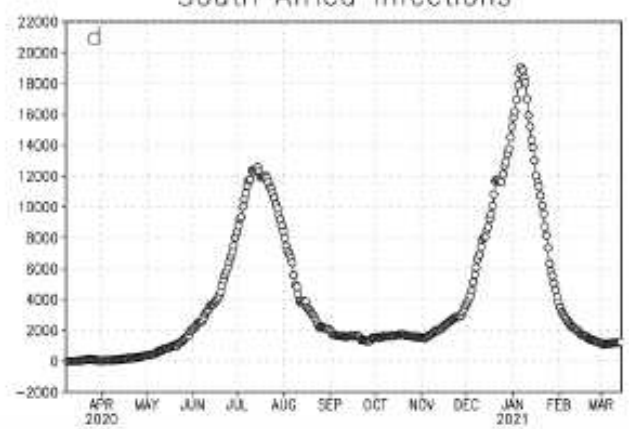

Western Cape deaths

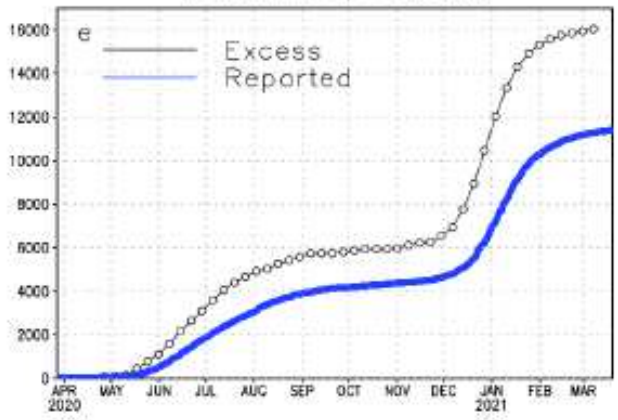

Eastern Cape deaths

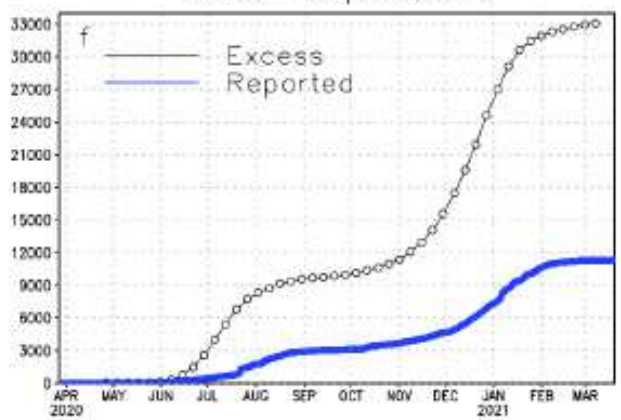

Gauteng deaths
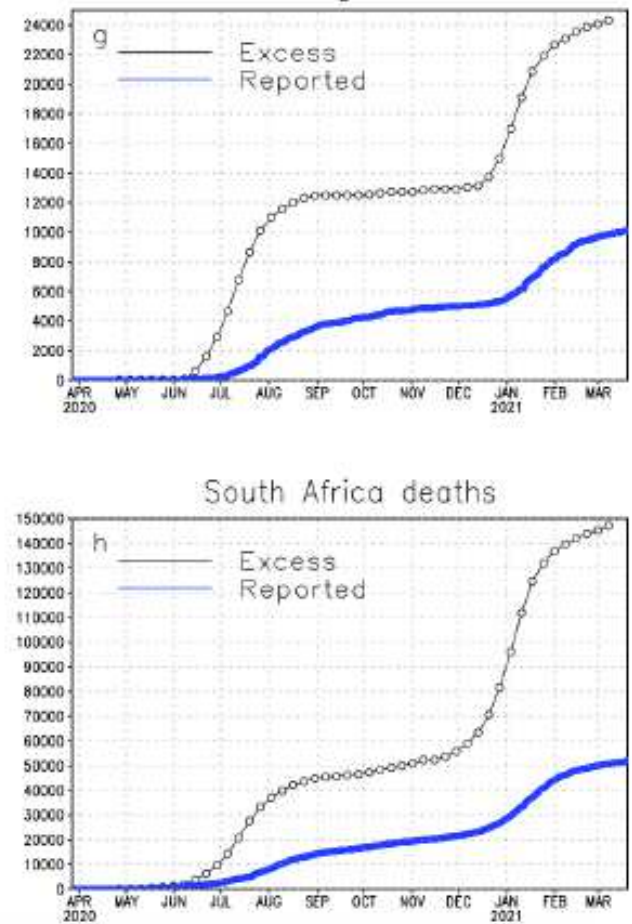

Figure 2

Left panels: Seven-day centered moving averages of daily reported Covid-19 infections for (a) Western Cape, (b) Eastern Cape, (c) Gauteng and (d) South Africa between 8 March 2020 and 14 March 2021. Right panels: Same as for the left panels, but showing accumulated excess (weekly, black lines) and reported (daily, blue lines) Covid-19 deaths through to 7 March and 19 March 2021, respectively. 



Figure 3

The reconstructed Covid-19 infections in the Gauteng province of South Africa for the period through to 7 March 2021 (black lines), and projected infections until the end of 2021 (blue and red lines), for an IFR of 0.31. Panel a represents the case where disease-induced immunity is ever lasting. Panel $b$ represents immunity lasting for 12 months. Panel c represents the case where immunity lasts for 12 months, but where the arrival of the new B.1.351 variant in late November 2020 was associated with $50 \%$ of the immune population at the time becoming susceptible to the new variant. Panel $d$ is similar to panel $b$, but with immunity lost after six months. For the projections it is assumed that NPI will restrict R0' to 1.4 through to 1 April 2021. The red lines represent a worst case scenario where R0' assumes the value as reconstructed for the second wave (Supplementary Table 3.1) from 2 April 2021 onwards. The blue lines represent a best-case scenario where NPI restricts $\mathrm{R} 0$ ' to 1.6 from 2 April onwards. 

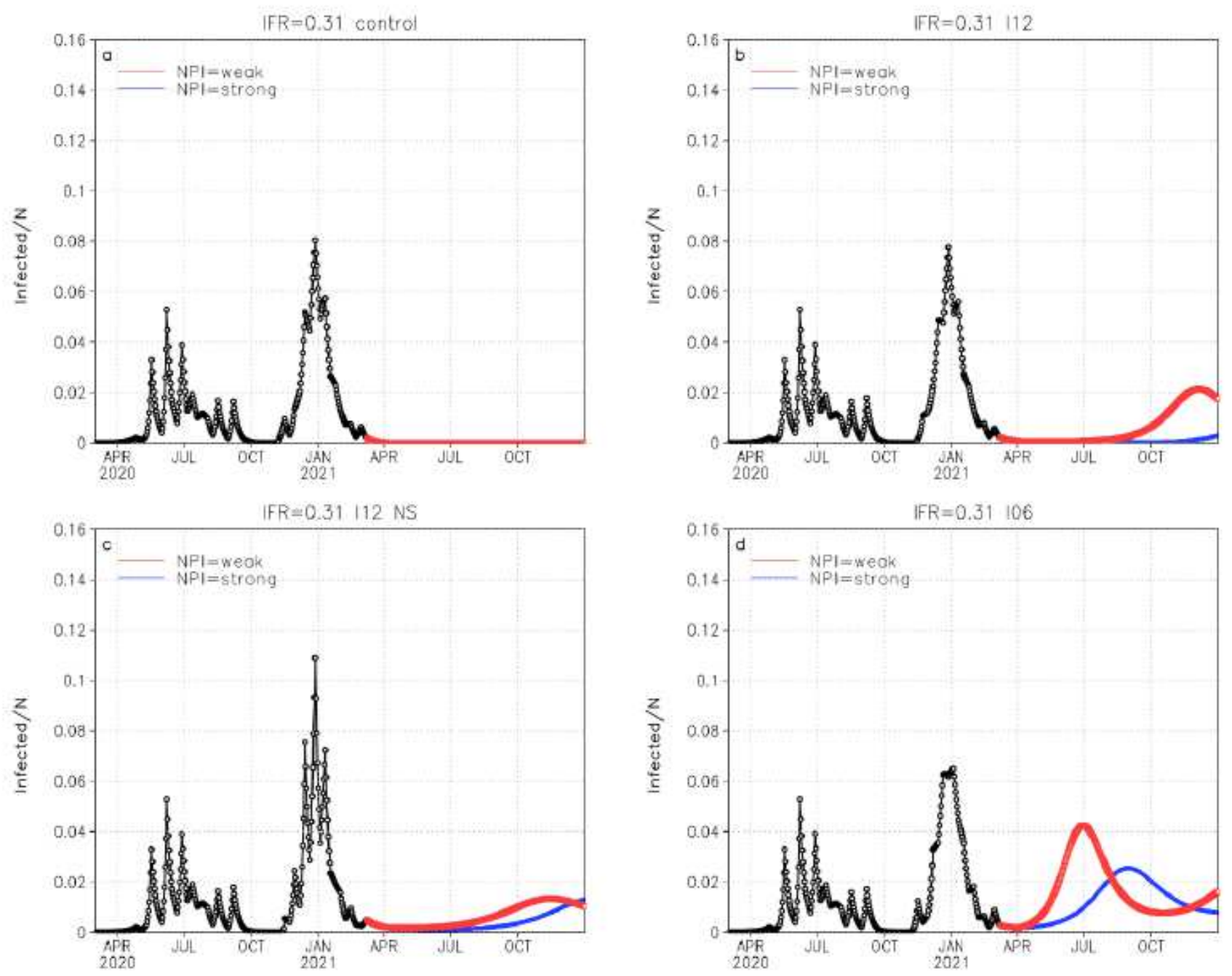

Figure 4

The reconstructed Covid-19 infections in the Western Cape province of South Africa for the period through to 7 March 2021 (black lines), and projected infections until the end of 2021 (blue and red lines), for an IFR of 0.31 . Panel a represents the case where disease-induced immunity is ever lasting. Panel $b$ represents immunity lasting for 12 months. Panel $c$ represents the case where immunity lasts for 12 months, but where the arrival of the new B.1.351 variant in early November 2020 was associated with $50 \%$ of the immune population at the time becoming susceptible to the new variant. Panel $d$ is similar to panel b, but with immunity lost after six months. For the projections it is assumed that NPI will restrict RO' to 1.4 through to 1 April 2021. The red lines represent a worst case scenario where R0' assumes the value as reconstructed for the second wave (Supplementary Table 3.1) from 2 April 2021 onwards. The blue lines represent a best-case scenario where NPI restricts R0' to 1.6 from 2 April onwards. 

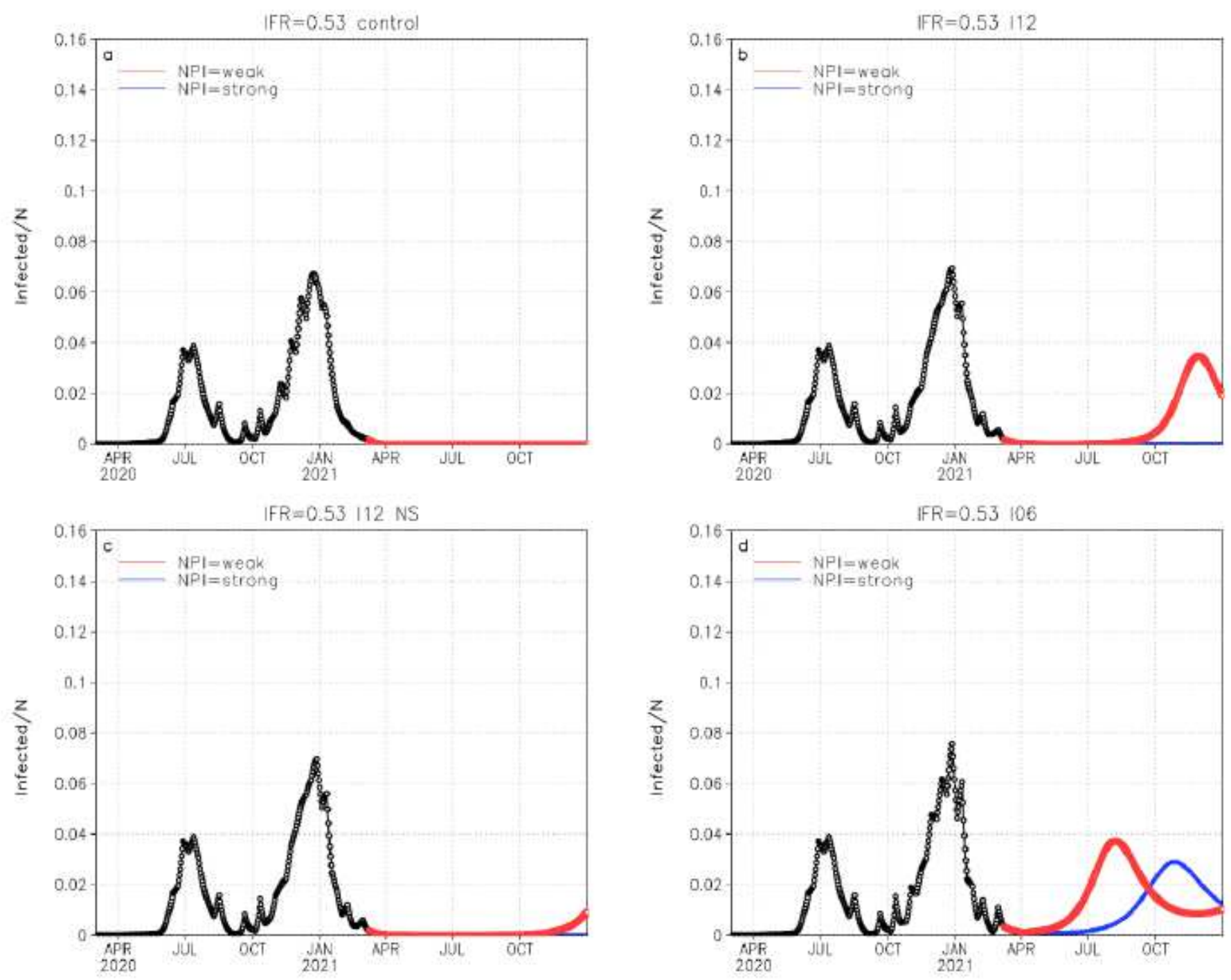

\section{Figure 5}

The reconstructed Covid-19 infections in the Eastern Cape province of South Africa for the period through to 7 March 2021 (black lines), and projected infections until the end of 2021 (blue and red lines), for an IFR of 0.53. Panel a represents the case where disease-induced immunity is ever lasting. Panel $b$ represents immunity lasting for 12 months. Panel $c$ represents the case where immunity lasts for 12 months, but where the arrival of the new B.1.351 variant in early October 2020 was associated with $50 \%$ of the immune population at the time becoming susceptible to the new variant. Panel $\mathrm{d}$ is similar to panel b, but with immunity lost after six months. For the projections it is assumed that NPI will restrict R0' to 1.4 through to 1 April 2021. The red lines represent a worst case scenario where R0' assumes the value as reconstructed for the second wave (Supplementary Table 3.1) from 2 April 2021 onwards. The blue lines represent a best-case scenario where NPI restricts Ro' to 1.6 from 2 April onwards. 